\title{
('ANCER AND SYPHILIS.
}

\author{
By GRIFFITH EVANS, M.A., D.M., F.R.C.S., Caernarvon.
}

THE object of a paper by Fry $(1930, \text { p. } 313)^{1}$ was "to ascertain ... the relation of syphilis to malignant disease as determined by the Wassermann reaction." The conclusion reached (p. 322) was: "In general, from the above figures, there is no evidence that syphilis plays any direct or very important part in the production of cancer."

This paper has been quoted by authoritative journals and the conclusion apparently accepted without regard to the fallacy of making the positive Wassermann reaction synonymous with syphilis.

The inadequacy of the Wassermann reaction as a diagnostic standard has been exposed so often that it is both surprising and regrettable to find it retaining a deciding value in any investigation such as Fry's, and especially that the conclusions based on it should be accepted without comment.

Kolmer (1923) ${ }^{2}$, in a series of 363 cases of latent syphilis, found a positive reaction in only 40 to 48 per cent.- the higher percentage being in the earlier stages. At the League of Nations Laboratory Conference in 1928, the Wassermann test was compared with certain flocculation tests. In 496 cases of syphilis a positive result was found in 208 only, and it is patent that for the purposes of this investigation no doubt as to the diagnosis was permissible. Again, it is well known that a negative Wassermann reaction is the rule in congenital lues after puberty.

When Farquhar Buzzard (1921) ${ }^{3}$ said that his faith in the Wassermann test as a diagnostic standard had been shattered, he expressed the only possible rational opinion.

But the foregoing is not the only ground for rejecting Fry's conclusions. They are contrary to clinical experience, and this experience would be more general if the signs of latent syphilis were better known. A learned profession, however, has allowed its judgment to be fettered by an empirical laboratory test and has shut its eyes to the large and increasing group of Wassermann negative syphilitics.

Force of circumstance compels the family practitioner to recognise and treat this class, and great is the reward of him who does so.

It is a matter of experience that malignant disease is more prevalent in this class than in any other. When I expressed this view to my former teachers

1 Fry, H. J. B. (1930). Syphilis and malignant disease. A serological study. J. Hygiene, 29, $313-22$.

${ }^{2}$ Kolmer, J. A. (1923). Infection, Immunity and Biological Therapy. 3rd ed. p. 571.

3 Buzzard, Farquhar (1921). Lancet, i, 65-7. 
it was said to be unacceptable because there was neither demonstrable evidence of the nature of the growth nor of the co-existence of syphilis. I have, therefore, attempted the necessary demonstration.

The number of cases to date is small but is consecutive and increasing. Only two are omitted because post-mortem examination was refused. The remaining twenty fall into three groups, in all of which malignancy was confirmed by microscopy.

1. Six cases where the co-existence of syphilis must be admitted on orthodox grounds.

2. Six cases where the orthodox will hesitate and will say either with one pathologist "the lesions are definitely pathological and might be syphilitic," or with another "I cannot say that the changes are syphilitic." Those, however, who accept and apply Warthin's standard of microscopical diagnosis will be more inclined to combine this with the clinical signs and agree that lues is present.

3. Eight cases where there is no evidence of syphilis.

If there were no positive correlation between the two diseases, the natural coincidence would be about 1 in 35 , assuming that 1 in 7 of the population is cancerous, and 1 in 5 syphilitic. The actual coincidence is, however, very much greater as clinicians, especially on the continent, have repeatedly stated.

\section{Group I.}

\section{Cases with both malignant disease and syphilis.}

1. Male. Carcinoma liver. Unequal Argyll-Robertson pupils. Absent knee jerks. Coronary thrombosis. Many miscarriages and no living children. W.R. ${ }^{1}$ negative.

2. Female. Carcinoma breast. Microscopical evidence of syphilitic aortitis. W.R. negative.

3. Female. Carcinoma breast. W.R. positive. Diagnosis of syphilis confirmed during stay in a teaching hospital.

4. Female. Carcinoma oesophagus. Wife of a syphilitic. Smooth pale area on either side of tongue. Knee jerks feeble. Ankle jerks absent. Plantar response extensor on both sides. Microscopical evidence of syphilitic aortitis.

5. Female. Carcinoma small intestine. W.R. positive (serum). C.S.F. ${ }^{2} 1 / 1000$ protein. Six lymphocytes per c.mm. W.R. positive (C.S.F.).

6. Female. Carcinoma rectum. W.R. negative. Multiple post-mortem evidence of syphilis unsuspected during life.

It is noteworthy that four of the foregoing cases had a negative Wassermann reaction (method No. 1 of Medical Research Council).

\section{Group II.}

Cases of malignant disease with circumstantial evidence of syphilis.

1. Male. Carcinoma stomach. Fibrosis and lymphocytic infiltration of both heart and liver. Wife is anaemic, has unequal pupils and smooth atrophic tongue. There were two children and two miscarriages. W.R. not done.

2. Male. Carcinoma rectum. Unequal pupils, paraesthesiae legs. Responded to antisyphilitic treatment before onset of malignancy. No children. Evidence of lues in several 
members of the wife's family. W.R. negative. Localised lymphocytic infiltrations in all organs examined.

3. Female. Carcinoma oesophagus. W.R., 1 M.H.D. ${ }^{1}$ Lymphocytic infiltration about vasa vasorum of aorta. Minor clinical signs of latent lues in self and good response to therapy in husband. No children.

4. Female. Carcinoma oesophagus. Long standing anaemia. Atrophic tongue, moist fissures angles of mouth. W.R., 1 M.H.D., C.S.F. $0 \cdot 3 / 1000$ protein. Positive therapeutic test before onset of malignancy. Post-mortem: fibrosis and cellular infiltrations in several organs on which a pathologist reported "definitely pathological and could be syphilitic."

5. Female. Carcinoma stomach. Hemiplegia of eight years' standing. Tongue red and glazed. One brother died of carcinoma stomach, another of ruptured aortic aneurism. Postmortem: localised perivascular infiltrations in liver and kidney.

6. Female. Cancer of breast 1921; cancer of uterus 1929. Both confirmed by microscopy. Unequal pupils. W.R., 1 M.H.D. Post-mortem: aorta, liver and pancreas show localised perivascular infiltrations of lymphocytes. The husband of this patient was one of four married brothers. No children of any marriage. One wife died of carcinoma stomach, another was found dead in bed. The remaining wife is healthy, but this brother has chronic intractable headache.

\section{GROUP III.}

Cases of malignant disease without evidence of syphilis.

1. Male. Carcinoma lung. Slight cellular fibrosis in heart and liver.

2. Female. Carcinoma uterus. Fatty cirrhotic liver. Pathologist's comment: "I cannot say that this is syphilitic."

3-8. Similar negative reports.

It is a matter of experience, confirmed in my opinion by the foregoing observations, that syphilis in man is a common pre-cancerous state. So common that the Spirochaete should rank with shale oil, etc., as a recognised cancer-producing irritant, and much more important because of its wide distribution. Shale-oil cancer is banished by prohibiting the use of the irritant, it is possible that the incidence of cancer in general could be diminished by the proper treatment of latent lues.

Many workers engaged in experimental cancer production in animals, and observers of occupational cancer in man, have stressed individual susceptibility. The majority of mule-spinners for example remain cancer free, the minority are more susceptible and develop cancer. It might be profitable to examine the latter for microscopical evidence of syphilis rather than to rely solely on the Wassermann test.

\section{Conclusion.}

Fry's investigation is shown to rest on the fallacy of assuming that all syphilitics have a positive Wassermann reaction.

Clinical evidence is given showing the co-existence of both cancer and syphilis in six out of twenty cases and their probable co-existence in six of the remainder.

1 M.H.D. = 1 minimum haemolytic dose of complement fixed (fixation of at least 3 M.H.D. necessary for a diagnosis of syphilis on serological grounds alone).

Journ. of Hyg. xxxiI 
General considerations Which ENTItLe SYPHILIS TO SPECIAL STUDY AS A PRE-CANCEROUS CONDITION.

It will be agreed that in man chronic irritation is the outstanding precancerous state, in other words that cancer is a disease of damaged tissue.

Clinicians fear that the logical sequence of animal experiments has led research workers into fields remote from human cancer, remote because of the extreme specificity of cancer. Clinical observations are written down as being of doubtful validity because they are incapable of direct proof. Examples will illustrate this. Ten years ago one of three sisters died of malignant disease of the spine-primary focus uncertain. Recently one died of heart failure having had right hemiplegia two years earlier. She had unequal pupils, a smooth patch on either side of the tongue, extensor plantar response on the left side, chronic arthritis both knees, painless on the left, anaesthesia of right ulnar trunk. In spite of the negative Wassermann reaction she represented in my opinion a definite case of chronic syphilis probably congenital. The third sister has a chronic dysphagia without organic obstruction.

The diagnosis of malignancy in the first sister, though obvious clinically, is of doubtful validity from the point of view of research; the diagnosis of syphilis in the second is also valueless as evidence because no autopsy was permitted. The occurrence of dysphagia in the remaining sister would appear to be of no importance whatever, but every one of ten cases of dysphagia in my experience has been thus related to syphilis and malignant disease, or has been cured by anti-syphilitic treatment.

Again, a man dies of carcinoma of the rectum, his brother bears stigmata of congenital lues. Another has carcinoma of the stomach and his wife has abdominal psoriasis with a positive Wassermann reaction. Instances of this kind could be multiplied, and in my experience there is an association of the two diseases in the majority of cases.

Believing therefore that experience pointed to a close inter-relation between syphilis and cancer, I turned to the literature. Here the outstanding features are, first, the complete disregard of both the signs of latent syphilis and of the microscopical standards of diagnosis laid down by Warthin (1927) ${ }^{1}$, and, second, the subjugation of clinical opinion to the Wassermann test. It will probably be agreed that cancer of the tongue would be extremely rare if there were no pre-existing syphilis.

The statistics of both the Registrar-General and the Medical Research Council's inquiry into occupational cancer show a definite correlation between cancer of the tongue, cirrhosis of the liver and cancer of the oesophagus. There is also an agreed but unexplained correlation between syphilis and cancer of the oesophagus. From the former figures Holmes $(1931)^{2}$ concludes that "the evidence makes it extremely probable that alcoholism is partly

1 Warthin, A. (1927). Cardio-vascular syphilis. Atlantic Med. J. (August).

${ }^{2}$ Holmes, B. (1931). Cancer and Scientific Research, pp. 39, 59. 
responsible for cancer in these organs (liver, tongue, oesophagus and stomach)." This conclusion is based on the statement that "In this country cirrhosis of the liver is practically always due to chronic alcoholism." It is true that alcohol is accepted as the cause of cirrhosis of the liver, but it is also true that its position is more due to tradition than to demonstration. When heavy drinking was universal and cirrhosis common it was not unnatural to associate the two, but efforts to produce cirrhosis in animals with concentrations of alcohol comparable with those ingested by man, have not afforded satisfactory evidence. On the other hand, we now know that syphilis was and is extremely prevalent and that every established syphilitic, whether congenital or acquired, has some degree of perivascular infiltration of the liver and that this may vary from slight plasma-celled infiltration of the periportal tissue to the most marked cirrhosis. So long, however, as the microscopical diagnostic standards of Warthin remain unaccepted, so long will alcohol retain its leading position in the etiology of cirrhosis, but those who accept these standards are likely to establish syphilis as the commonest cause of cirrhosis and at the same time to add to our knowledge of the pre-cancerous state.

I will again quote Holmes (1931): "On the whole the most generally accepted belief at the moment and one which has convincing figures from Sumatra and Calcutta to support it, is that liver cancer supervenes on cirrhosis of the liver-which is a common disease among the natives. In Sumatra a very large percentage occurs in definitely cirrhotic livers."

Mohammedans abstain from alcohol, but they share the liability to cirrhosis and carcinoma, and no hypothesis can be true unless it is applicable to all the known facts.

In ten years I have attended five cases of carcinoma of the liver (so called because no primary focus was evident). One had a negative Wassermann but was syphilitic, three had a positive Wassermann reaction with additional clinical evidence, and the family of the last is notorious for chronic degenerative conditions.

With regard to cancer of the oesophagus, I have (1930) ${ }^{1}$ elsewhere attempted to show that the group of dysphagias, which includes achalasia, constitute manifestations of latent lues. The liability to malignant change in this group has been repeatedly emphasised. Further, the correlation between syphilis and cancer of the gullet revealed in the reports of the Registrar-General still awaits an explanation.

It will be realised that the difficulty in proving a direct connection between cancer and syphilis, especially the inherited type, lies in the diagnostic obscurity into which sufferers from the latter gradually recede as the infection becomes less and less virulent.

Warthin thus expresses the difficulty: "Undoubtedly the clinician of the present day has lost much skill in the diagnosis of syphilis; he does not know the pathology of the disease sufficiently well as to be always on the look-out

1 Evans, G. (1930). Practitioner, p. 317. 
for its protean manifestations and especially because he has come to depend mainly on one thing - the laboratory report of a negative or positive Wassermann reaction."

It is evident that an individual once infected with the Spirochaete, congenitally or otherwise, has within him multiple foci of chronic inflammation, in some of which active tissue proliferation persists throughout life.

It is not for the clinician to say where the line separating the normal from malignant proliferation is transgressed, but that cancer may thus result is undoubted. Workers like Arthur Eastwood will ultimately show how and why the change occurs.

If, however, it is possible to demonstrate a close, not necessarily constant, association between cancer and syphilis a decided advance will be made, and this is a demonstration which only the family practitioner working over a long period of years is qualified to make.

(MS. received for publication 14. v. 1931.-Ed.)

\section{Editorial Note.}

We have published Dr Evans' paper because the question raised is of interest, but, particularly in view of the lamented death of Dr Fry, it is necessary to point out that the data here given do not make out even a prima facie case in favour of the suggestion that syphilis and cancer are aetiologically related. If Dr Evans' suggestion were correct that of all persons who come to autopsy at the cancer age 1 in 7 will be cancerous and 1 in 5 syphilitic, then on the average 1 in 35 of all the cadavera will present the stigmata of both cancer aud syphilis. Dr Evans is not offering such a random sample, but a set of 20 cases in all of which cancer was found, naturally, by hypothesis, 1 in 5 of these should present the stigmata of syphilis, or 4 out of the 20. The observed number 6 differs from the expected number by little more than the standard error in the latter. 